\title{
Symptoms and signs of progressive hydrocephalus
}

\author{
M KIRKPATRICK, H ENGLEMAN, AND R A MINNS \\ Department of Neurology, Royal Hospital for Sick Children, Edinburgh
}

SUMmARY The clinical features of 107 cases of children with hydrocephalus and measured raised intraventricular pressure were analysed retrospectively. Fifty one children had recently been diagnosed as having hydrocephalus, and the remainder had had shunts injected to direct the cerebrospinal fluid. The most common symptoms in the group were vomiting, behavioural changes, drowsiness, and headaches. The most common clinical signs were inappropriately increasing occipitofrontal head circumferences, tense anterior fontanelles, splayed sutures, and distension of the scalp veins. Half the infantile cases of hydrocephalus were without symptoms, and a quarter of the cases with cerebrospinal fluid shunts and measured raised intraventricular pressure were without signs. There were no fewer than 33 different clinical signs including several unusual ones, such as macular rash and sweating. We believe that the presentation of hydrocephalus with raised intraventricular pressure is sufficiently variable, unusual, or even absent to justify the direct measurement of intracranial pressure.

The classical adult presentation of raised intracranial pressure (headache, vomiting, and papilloedema $^{1}$ ) is rare in children with progressive hydrocephalus. There are few studies of the clinical presentation of hydrocephalus with raised intracranial pressure either before or after an operation to divert the cerebrospinal fluid.

There are certainly no studies in which raised intracranial pressure has been confirmed by direct measurement, and such reports that do exist in children frequently include those with causes of raised intracranial pressure other than hydrocephalus alone. The aim of this study therefore was to document the symptoms and signs of 107 cases of progressive hydrocephalus (that is, hydrocephalus with raised intracranial pressure) in which the clinical features were documented and raised ventricular pressure had been confirmed on direct measurement.

\section{Patients and methods}

All the patients were admitted to the neurology unit at the Royal Hospital for Sick Children, Edinburgh, from 1975 onwards and they all had progressive hydrocephalus with or without a ventriculoperitoneal shunt in situ. Patients who had raised intracranial pressure from encephalopathies due to brain swelling or cerebrovascular congestion, or intracerebral space occupying lesions, were ex- cluded (even if there was also hydrocephalus). Those with ventriculitis were also excluded. Table 1 shows the underlying aetiologies in the 107 cases. Hydrocephalus associated with spina bifida and idiopathic hydrocephalus were the most common presentation $(65 \%)$.

Fifty one of the 107 cases had clinical features and a documented recording of raised intracranial pressure before any procedure to divert the cerebrospinal fluid was undertaken ( 32 boys and 19 girls). Fifty six cases presented with raised intracranial pressure and ventriculoperitoneal shunts in situ, the clinical features thus representing shunt malfunction (29 boys and 27 girls). Fifty one were infants ( 44 of the non-shunted and seven of the shunted), 22 cases were between the ages of 1 and 4 (two of the nonshunted and 20 of the shunted), and 34 cases were aged 5 or over (five of the non-shunted and 29 of the shunted).

Table 1 Original aetiology of hydrocephalus

\begin{tabular}{lc}
\hline Diagnosis & $N o$ \\
\hline Hydrocephalus associated with spina bifida complex & 58 \\
Idiopathic & 14 \\
Post haemorrhagic hydrocephalus & 14 \\
Post meningitic hydrocephalus & 11 \\
Aqueduct stenosis & 8 \\
Hydranencephaly & 1 \\
Congenital toxoplasmosis & 1 \\
\hline
\end{tabular}


The data that were recorded included name, age, sex, the measured intracranial pressure, the duration of symptoms and signs before the raised intracranial pressure was diagnosed, the symptoms and signs themselves, and whether or not a cerebrospinal fluid shunt had already been injected. If hydrocephalus and raised intracranial pressure (in the absence of any other cause for raised pressure) were confirmed on admission, the pro forma was completed and the data were analysed retrospectively.

The method of measurement of intracranial pressure varied; for those who presented in infancy without a shunt, it was measured percutaneously through the anterior fontanelle or through a Rickham ventriculostomy reservoir that had been inserted as an elective procedure. For those patients with ventriculoperitoneal shunts in situ, the pressure was measured through separate ventriculostomy reservoirs. It has been our practice to insert a subcutaneous cerebrospinal fluid reservoir at the same time as the shunt, thus enabling us to measure the intracranial pressure directly whenever there was a suspicion of shunt malfunction. ${ }^{2}$ In all cases a Gaeltec miniature strain gauge transducer was used and a direct recording of an opening pressure, maximum resting pressure, or mean overnight pressure was obtained.

\section{Results}

The most common presenting symptoms and signs of progressive hydrocephalus are shown in table 2 . This group comprises both early hydrocephalus in non-shunted children and those with shunt malfunction.

The maximum sustained intracranial pressure measured in these cases was between 10 and $20 \mathrm{~mm}$ $\mathrm{Hg}$ in $12(11 \%)$, between 20 and $29 \mathrm{~mm} \mathrm{Hg}$ in 29 $(27 \%)$, and over $30 \mathrm{~mm} \mathrm{Hg}$ in $60 \%$ of cases. There was no correlation between any clinical feature and the measured intracranial pressure.

Fifty eight had symptoms and signs for less than two weeks before the diagnosis of raised intracranial pressure was made $(54 \%)$. Twenty one $(20 \%)$, however, had symptoms and signs for longer than one month and this group includes six cases who had symptoms and signs for more than three months before diagnosis.

The 51 cases of progressive hydrocephalus before surgical treatment have a different range of symptoms and signs from the total group (table 3 ). This group, as would be expected, contains many more younger children and half of these with measured raised intracranial pressure were asymptomatic.

The second subgroup (56 cases with shunt mal- function) is shown in table 4. They are older than the first subgroup, and their signs are even more varied with no obvious signs on clinical examination in a quarter.

Table 2 Most common clinical features of progressive hydrocephalus, both shunted and non-shunted

\begin{tabular}{lc}
\hline & No (\%) \\
\hline Symptoms: & \\
Vomiting & $35(33)$ \\
Behaviour change (including irritability) & $32(30)$ \\
Drowsiness or lethargy & $30(28)$ \\
Headache & $30(28)$ \\
Asymptomatic & $30(28)$ \\
Anorexia & $15(14)$ \\
Stiff neck or back ache & $8(7)$ \\
Signs: & \\
Inappropriately increasing occipitofrontal & $43(40)$ \\
circumference & $33(31)$ \\
Tense anterior fontanelle & $22(21)$ \\
Splayed cranial sutures & $19(17)$ \\
Scalp vein distension & $16(15)$ \\
Sunsetting or loss of upward gaze & $15(14)$ \\
No signs & $14(13)$ \\
Decreased conscious level & $13(12)$ \\
Neck retraction or rigidity & $11(10)$ \\
Acute strabismus & $9(8)$ \\
Pupillary changes & $8(7)$ \\
Papilloedema & $8(7)$ \\
Distended retinal veins & \\
\hline
\end{tabular}

Other less frequent symptoms were: jitteriness, sleep disturbance, sluggish valve, fits, abdominal pain, deteriorating school performance, pallor, and failure to thrive. Other less frequent signs were: tonic fits, pallor, sluggish valve, decerebration, optic atrophy, nystagmus, bradycardia, hypertension, profuse sweating, neurogenic stridor, macular rash, return of tonic neck reflex, hemiparesis, ataxia, sudden blindness, Cheyne-Stokes respiration, apnoeic or cyanotic episodes, cardiorespiratory arrest, ptosis, VII and XII cranial nerve palsies, and vasomotor changes.

Table 3 Most common clinical features of progressive hydrocephalus in the non-shunted group $(n=51)$

\begin{tabular}{lr}
\hline & No (\%) \\
\hline Symptoms: & \\
Asymptomatic & $25(49)$ \\
Headache or irritability & $17(33)$ \\
Vomiting & $8(16)$ \\
Signs: & \\
$\quad$ Inappropriately increasing occipitofrontal & \\
$\quad$ circumference & $39(76)$ \\
Tense anterior fontanelle & $33(65)$ \\
Splayed sutures & $20(39)$ \\
Scalp vein distension & $17(33)$ \\
Sunsetting or loss of upward gaze & $11(22)$ \\
Neck retraction or rigidity & $7(14)$ \\
\hline
\end{tabular}

Other symptoms (in 5\%-10\% of cases): jittery (infants), anorexia, drowsiness or lethargy, and stiff neck. Other signs (in $5 \%-10 \%$ of cases): decreased conscious level, pupillary changes, neurogenic stridor, decerebration, and papilloedema. 
Table 4 Most common clinical features of progressive hydrocephalus in the shunted group $(n=56)$

\begin{tabular}{lr}
\hline & No (\%) \\
\hline Symptoms: & \\
Vomiting & $27(48)$ \\
Drowsiness or lethargy & $26(46)$ \\
Headache & $26(46)$ \\
Behaviour change (including irritability) & $21(38)$ \\
Anorexia & $10(18)$ \\
Signs: & \\
No clinical signs & $14(25)$ \\
Decreased conscious level & $10(18)$ \\
Acute strabismus & $10(18)$ \\
Neck retraction & $6(11)$ \\
Distended retinal veins & $6(11)$ \\
\hline
\end{tabular}

Other symptoms (in $5 \%-10 \%$ of cases): valve not working, sleep disturbance, stiff neck, asymptomatic, and fits. Other signs (in $5 \%-10 \%$ of cases): sluggish valve, sunsetting, papilloedema, tonic fits, pallor, inappropriately increasing occipitofrontal circumference, tense anterior fontanelle, optic atrophy, bradycardia, profuse sweating, nystagmus, hypertension, and macular rash.

\section{Discussion}

There are two distinct groups of patients with progressive hydrocephalus in this study: those with and those without diverting shunts. Those with shunts and raised intracranial pressure, therefore, have clinical features representative of shunt malfunction, that is blockage or disconnection.

We were not concerned with arrested hydrocephalus, or with ventriculomegaly resulting from atrophic or ischaemic parenchymal damage as may be seen, for example, in preterm infants with periventricular cystic leukomalacia; they do not have raised intracranial pressure and therefore do not fulfil the entry criteria for this study. Nor were we concerned with other causes of raised intracranial pressure in children such as cerebral oedema or space occupying lesions (even if dilated ventricles were a symptom), as in those cases the clinical features may be a reflection of the disease itself. Tadros et al reported 100 adult cases of raised intracranial pressure that had the clinical features of headache, papilloedema, disturbance of consciousness, vomiting, optic atrophy, false localising signs, and fits (in decreasing order of frequency). ${ }^{3}$ Most of these patients, however, had intracranial tumours.

\section{MOST COMMON SYMPTOMS}

Vomiting-is a non-specific symptom in childhood (and may therefore be a reason for late presentation or delay in diagnosis). It was the commonest presenting symptom in the shunted group $(n=27)$ and the third most frequent presentation in the nonshunted group $(\mathrm{n}=8)$.

Behavioural change-including irritability-was the second most frequent symptom in the whole group $(n=32)$. It seemed to be a frequent presentation of both subgroups and does not happen only in those with closed sutures, but at all ages. Irritability in infants may have reflected headaches but, like vomiting, is a non-specific symptom.

Headache-was more common in the older shunted group $(n=26)$. The headache associated with raised intracranial pressure classically occurs after waking from a phase of rapid eye movement sleep when there is increased cerebral blood flow and a nocturnal rise in intracranial pressure. ${ }^{4}$ In the series of intracranial tumours reported by Tadros et al, headaches were reported in all the patients. ${ }^{3}$

Anorexia-occurred in 15 cases (14\%). This may, together with vomiting and a long duration of symptoms before diagnosis of raised intracranial pressure, have been the reason for the failure to thrive of one case in this series.

Many of the symptoms of raised pressure (vomiting, behavioural changes, drowsiness, headaches), are similar to symptoms of upper respiratory infection. The reasons for this may be firstly, that children with upper respiratory infection have increased cerebral blood flow and in a hydrocephalic child with abnormal cerebrospinal fluid dynamics this may result in a rise in intracranial pressure. Secondly, some cerebrospinal fluid may be absorbed through the paranasal sinuses ${ }^{5}$ and therefore a rise in intracranial pressure may lead to facial congestion, headache, and so on-similar to an upper respiratory illness.

\section{MOST COMMON CLINICAL SIGNS}

Inappropriately increasing occipitofrontal circumference - was the most common sign in the nonshunted group and occurred in $39(76 \%)$ of cases, all of whom were infants. This was a presenting sign, however, in only four cases (also infants) in the shunted group. Dandy in 1920 considered that all the symptoms and signs accompanying hydrocephalus were the direct result of pressure exerted by the cerebrospinal fluid. ${ }^{6}$ Though an increasing head circumference is usually a direct effect of raised intracranial pressure, there may be a secondary effect of intracranial venous obstruction leading to an increased choroid plexus capillary pressure and a subsequent further rise in cerebrospinal fluid production. ${ }^{7}$ Thus the expanding head circumference is compensatory and acts as a buffer.

$A$ tense anterior fontanelle-was the second most common finding in the whole group $(n=33,65 \%)$. In conditions of raised intracranial pressure the 
normally concave and pulsatile fontanelle becomes tense, and at extremely high pressure loses its pulsatility.

Splayed sutures were the third most common clinical sign and were present in 20 cases in the nonshunted group and two (both infants) in the shunted group. The clinical features clearly depend on whether the raised intracranial pressure occurs in an expansile or non-expansile head. Sutural closure begins before birth with fusion of the metopic sutures and progresses through posterior and anterior fontanelle closure up to age of 12 years when it is thought that raised intracranial pressure can no longer separate the sutures.

Distended scalp veins-were present in a third of the cases in the non-shunted group. These are usually particularly prominent in the frontal and temporal regions. Spalteholz valves are situated between the intracranial and extracranial veins, and allow a unidirectional flow to the outside. Part of the cerebral blood flow is therefore drained by these and raised intracranial pressure is transmitted to them.

'Sunsetting'-(an inability to look upwards, found mostly in infants) may initially be intermittent but later becomes continuous, and is due to pressure on the superior quadrigeminal plate against the free edge of the tentorium. There is, however, a complex of signs resulting from lesions in this area known as the Koerber-Salus-Elschnig sylvian aqueduct syndrome. ${ }^{8}$ This consists of paralysis of upward gaze, pupillary abnormalities, abnormalities of convergence and nystagmus. Sunsetting, pupillary changes, and nystagmus occurred in $30(28 \%)$ of all cases.

Absence of symptoms-in the whole group there was an absence of symptoms despite the presence of clinical signs in $30(28 \%)$. In the non-shunted group there were 25 patients who were symptom free $(49 \%)$, all of whom were infants. Thus in infants without a shunt, progressive hydrocephalus was asymptomatic in almost half the cases, but by contrast only $7 \%$ of the shunted group were symptom free.

Absence of clinical signs in the presence of symptoms, however, occurred in $14 \%$ of the whole group and was the most frequent presentation in the shunted group $(25 \%)$. We believe that this supports the case for the direct measurement of the intracranial pressure, because one cannot rely on clinical features to make the diagnosis.

Decreased conscious level-14 cases in the whole group had a decrease in conscious level $(13 \%)$, four in the non-shunted and ten in the shunted group. It is generally assumed that coma is an infrequent presentation of hydrocephalus, but lesser degrees of impaired consciousness are not uncommon. Coma can occur with concomitant plateau waves on the intracranial pressure tracing, and may complete the clinical Charcot triad of pupillary dilatation and ipsilateral hemiplegia in the course of tentorial herniation as a complication of acute hydrocephalus.

\section{UNUSUAL CLINICAL FEATURES}

Neurogenic pulmonary oedema from a sudden rise in intracranial pressure was described by Ducker, ${ }^{9}$ who found that he could reproduce it experimentally in animals in several different ways. The bobble head doll syndrome caused by dilatation or colloid cysts of the third ventricle consists of $2-3 \mathrm{~Hz}$ oscillatory movements of the head and developmental delay. ${ }^{10}$ No child in this series had either pulmonary oedema or the full syndrome.

Profuse sweating occurred with raised intracranial pressure in four cases in all. The original description of diencephalic autonomic epilepsy by Penfield in $1929^{11}$ included profuse sweating as one of the features, but some authors have speculated that this was as a result of raised intracranial pressure. ${ }^{12} \mathrm{We}$ have documented some previously unreported signs-for example, a macular rash occurred in three cases on the extensor surfaces of the limbs at the same level of intracranial pressure on each occasion.

Ptosis is also an unusual presentation and autonomic dysfunction such as abdominal pain and pallor were more common than we expected.

Neurogenic stridor was present in four cases, all of whom were infants before shunting. This represents deranged lower brain stem function caused by bilateral cortico-bulbar disruption, and may occur alone or with other features of pseudobulbar palsy. ${ }^{13}$ Stridor may also occur after acute ventricular decompression of cerebrospinal fluid with an accompanying upward cone. Other evidence of pseudobulbar paresis included sucking and feeding difficulties, although frequently hydrocephalic infants with seriously raised intracranial pressure will feed and suck well. Difficulties with phonation, drooling, regurgitation, and recurrent aspiration pneumonia may also result from brain stem dysfunction.

It is of interest that papilloedema occurred in only eight cases in the whole group (8\%), two of whom were infants. In infantile hydrocephalus papilloedema occurs less often than stridor, pupillary changes, and diminished level of consciousness. This is in pronounced contrast to patients with infratentorial and supratentorial tumours among whom the incidence of papilloedema is $70 \%$ and $50 \%$, respectively. ${ }^{14}$ 


\section{CHRONIC SYMPTOMS}

The chronic symptoms of presentation of hydrocephalus and raised intracranial pressure must be distinguished from the clinical features of arrested hydrocephalus. The latter include ataxic and spastic cerebral palsy, ${ }^{15}$ hypothalamic and pituitary disturbance (for example, precocious puberty), ${ }^{16-18}$ mental retardation, and specific learning disorders. ${ }^{19}$ Although we encountered some patients with acute hemiplegia or ataxia, these signs all reverted to normal after relief of the raised intracranial pressure. $^{20}$

Twenty per cent of cases had had features present for more than one month, and six cases for more than three months. The clinical features that we recognised in those with a long duration of presentation were an insidious deterioration in school performance, intermittent headaches over many months, behavioural changes; failure to thrive, and dizziness. Many of these features resolved after relief of cerebrospinal fluid pressure.

In conclusion, therefore, we have shown the variability, unreliability, unusual nature, and even absence of the clinical features of hydrocephalus with raised intracranial pressure. Because imaging is a static investigation, and does not invariably indicate the degree or even the presence of raised intracranial pressure, we feel that our results highlight the need for direct measurement of intracranial pressure as the only sure way to establish its presence. Any unexplained clinical features in children with a cerebrospinal fluid shunt should alert the physician to the possibility of raised pressure from shunt malfunction.

We thank Action Research for the Crippled Child for continued support of this research.

\footnotetext{
References

${ }^{1}$ Bannister R, ed. Brain's clinical neurology. 6th ed. Oxford: Oxford University Press, 1985:229.

${ }^{2}$ Leggatt JRS, Baxter P, Minns RA, et al. Role of a separate subcutaneous cerebrospinal fluid reservoir in the management of hydrocephalus. Br J Neurosurg 1988;2:327-38.
}

${ }^{3}$ Tadros FW, Serour H, Zaki SA, Sakr R. The so-called general symptoms of increased intracranial pressure. Postgrad Med J 1962;38:417-6.

${ }^{4}$ Cooper R, Hulme A. Intracranial pressure and related phenomena during sleep. J Neurol Neurosurg Psychiatry 1966;29: 564-77.

5 Weed LH, McKibben PS. Pressure changes in the cerebrospinal fluid following intravenous injections of solutions of various concentrations. Am J Physiol 1919;48:512-30.

6 Dandy WE. The diagnosis and treatment of hydrocephalus resulting from strictures of the aqueduct of Sylvius. Surg Gynecol Obstet 1920;31:340-58.

7 Anderweg J. The cause of hydrocephalus. Rotterdam: BronderOffset BV, 1976:266-8.

${ }^{8}$ Chattha AS, DeLong GR. Sylvian aqueduct syndrome is a sign of acute obstructive hydrocephalus in children. $J$ Neurol Neurosurg Psychiatry 1979;38:288-96.

${ }^{9}$ Ducker TB. Increased intracranial pressure and pulmonary edema. 1. Clinical study of 11 patients. J Neurosurg 1968;28: $112-7$.

10 Tomasovic JA, Nellhaus G, Moe PG. The bobble-head doll syndrome: an early sign of hydrocephalus. Dev Med Child Neurol 1975;17:777-83

1 Penfield W. Diencephalic autonomic epilepsy. Arch Neurol 1929;22:358-74.

12 Summers DG, Young AC, Little RA, Stoner HB, Forbes WSTC, Jones RAC. Spontaneous periodic hypothermia with lipoma of corpus callosum. J Neurol Neurosurg Psychiatry 1981;44:1094-9.

13 Adeloye A, Singh SP, Odeku EL. Stridor, myelomeningocele and hydrocephalus in a child. Arch Neurol 1970;23:271-3.

14 Bell WE, McCormick WF. Symptoms, signs and physiologic concepts. In: Increased intracranial pressure in children. 2nd ed. Philadelphia: WB Saunders, 1978:7.

15 Hagberg B, Sjorgen I. The chronic brain syndrome of infantile hydrocephalus. Am J Dis Child 1966;112:189-96.

${ }^{16} \mathrm{Kim}$ CS, Bennett DR, Roberts TS. Primary amenorrhoea secondary to non communicating hydrocephalus. Neurology 1969;19:533-5.

${ }^{17}$ Fiedler R, Krieger DT. Endocrine disturbances in patients with congenital aqueduct stenosis. Acta Endocrinol 1975;80:1-13.

${ }^{18}$ Hier DB, Wiehl AC. Chronic hydrocephalus associated with short stature and growth hormone deficiency. Ann Neurol 1977;2:246-8.

19 Lonton AP. The relationship between intellectual skills and the computerised axial tomograms of children with spina bifida and hydrocephalus. $Z$ Kinderchir 1979;28:368-74.

${ }^{20}$ Bolthauser E, Hirsig J, Isler W, Rickham PP. Hemiparesis: an uncommon symptom of hydrocephalus or shunt dysfunction. $Z$ Kinderchir 1980;30:191-7.

Correspondence and requests for reprints to $\mathrm{Dr} R \mathrm{~A}$ Minns, Department of Neurology, Royal Hospital for Sick Children, Edinburgh EH9 1LF.

Accepted 26 April 1988 